\title{
Quantitative Analysis on Robustness of FLD and PCA- based Face Recognition Algorithms
}

\author{
Mahbuba Begum \\ Department of CSE \\ Mawlana Bhashani Science \\ and Technology University, \\ Tangail-1902, Bangladesh
}

\author{
Md. Golam Moazzam \\ Department of CSE \\ Jahangirnagar University \\ Savar, Dhaka-1342, \\ Bangladesh
}

\author{
Mohammad Shorif Uddin \\ Department of CSE \\ Jahangirnagar University, \\ Savar, Dhaka-1342, \\ Bangladesh
}

\begin{abstract}
Principal Component Analysis (PCA) has emerged as a more efficient approach for extracting features for many pattern classification problems. It has been the standard approach to reduce the high-dimensional original pattern vector space into low-dimensional feature vector space, that removes some of the noisy directions. PCA is an unsupervised technique which does not include label information of the data. In addition to PCA, another method Fisher Linear Discriminant (FLD) analysis has been widely used. In this paper, we report experimental results to quantify the robustness of PCA and FLD methods for face recognition. The experimentation was performed based on different levels of additive noise and rotations in handling face recognition problem. FLD outperforms the traditional PCA on the basis of robustness.
\end{abstract}

\section{Keywords}

Eigenface, Fisherface, Eigenvector, Featurevector, Fisher Linear Discriminant, Image Noise.

\section{INTRODUCTION}

A face recognition system is a computer technology. It identifies or verifies a person from a digital image or a video frame from a video source without direct human intervention. It is done by comparing selected facial features from the image and a facial database. Face recognition has many real world applications, like human-computer interface, surveillance systems, video-conferencing, forensic applications, pedestrian detection, image databases, etc. However, the development of a reliable system for human face recognition in a complex scene is very difficult due to variation in illumination, variability in scale, location, orientation (up-right, rotated) and pose (frontal, profile). Furthermore, facial expression, occlusion and lighting conditions change the overall appearance of face.

The face recognition problem has been faced up with various approaches over the last few decades: neural network, principal components, independent components, skin color based methods [1], [2]. Each of them imposes some constraints: frontal view, expressionless images, limited variations of lighting conditions, hairstyle dependence, uniform background, and so on. Yokoo et al. [3] proposed a face detection method by searching an ellipse using the genetic algorithm. As the performance of edge detection is quite dependent on lighting conditions, so an ellipse may not occur in a true face. Yang et al. [4] utilized a hierarchical knowledge-based system which consists of three levels of detection. Level 1 and level 2 are based on mosaic images, so the method is difficult to locate face regions accurately.
Furthermore, these methods are unable to detect a rotated human face.

The main objective of this work is to report experimental results to quantify the robustness of PCA and FLD methods in tackling noise and rotation for face recognition problem. Experimental results demonstrate that FLD outperforms the traditional PCA on the basis of robustness.

This paper is structured as follows. Section 2 describes PCA based face recognition. Section 3 explains FLD based face recognition. Section 4 presents the experimental results. Section 5 analyses the performance. Finally, Section 6 concludes the paper.

\section{PCA BASED FACE RECOGNITION}

Principle Component Analysis is one of the simplest and straightforward approach of reducing dimension. It takes multiple face images as input and finds eigenvectors of the covariance matrix. Then, it throws the earliest data onto a lower dimensional feature space, which is defined by eigenvectors with large eigen values. In this case, calculated eigenvectors are referred to as eigenfaces. This eigenface approach finds the lower dimensional space efficiently. This approach uses PCA [5], [6] for reducing dimension. It maximizes the total scatter across all classes of images. This method is best for reorganization of images from a lowdimensional basis.

PCA reduces dimension without losing too much information. It calculates eigenvectors which are non-zero vectors. The mathematical equation is as follows:

$$
A v=\lambda v
$$

Where $A$ is a square matrix, $v$ is an eigenvector of $A$ which is a non-zero vector. $\lambda$ is a scalar which is the eigenvalue of $A$ corresponding to $v$. The set of all eigenvectors with the same eigenvalue together with the zero vector is known as eigenspace. Eigenfaces are a set of eigenvectors [7], [8] used in the computer vision problem of human face recognition. The eigenfaces are the important components of face recognition. In image processing, processed images of faces can be considered as vectors whose components are the brightness of each pixel.

\subsection{Basic Steps Employed in PCA}

Step 1: Construct a face database that consists of the face images.

Step 2: Choose a training database that includes a number of images $(M)$ for each person with different degrees of rotation and different density of noise. 
Step 3: Calculate the $M \times M$ matrix $L$. Then find its Eigenvectors and Eigenvalues, and choose the $M^{\prime}$ Eigenvectors with the highest associated Eigenvalues.

Step 4: Combine the normalized training set of images to produce $M^{\prime}$ Eigenfaces.

Step 5: Store these Eigenfaces for later retrieval.

Step 6: For each member in the face database, compute and store a feature vector.

Step 7: Choose a threshold value $f$ that defines the maximum allowable distance from any face class.

Step 8: For each new face image to be identified, calculate its feature vector and compare it with the stored feature vectors.

Step 9: If the comparison satisfies the threshold for at least one member, then classify this face image as "known", otherwise a miss has occurred and classify it as "unknown" and add this member to the face library with its feature vector.

\section{FISHER LINEAR DISCRIMINANT BASED FACE RECOGNITION}

Fisher Linear Discriminant (FLD) has recently emerged as a more efficient approach for extracting features for many pattern classification problems as compared to traditional PCA. FLD produces well separated classes in a lowdimensional subspace, even under severe variation of rotating and noisy expressions [9]. The Eigenface technique, another method based on linearly projecting the image space to a low dimensional subspace, has similar computational requirements. An experimental analysis on the performances of FLD and traditional PCA were performed in tackling noise and rotation in face recognition problem with the face database. The experimental results show that FLD outperforms the traditional PCA on the basis of robustness. Fisherface is one of the efficient face recognition methods. However, Fisherface requires several training images for each face, so it cannot be applied to the face recognition applications where only one example image per person is available for training [10], [11].

FLD is an example of a class specific method, in the sense that it tries to shape the scatter in order to make it more reliable for classification. This method selects $W$ in such a way that the ratio of the between-class scatter and the withinclass scatter is maximized.

Let the between-class scatter matrix be defined as:

$$
S_{B}=\sum_{i=1}^{c} N_{i}\left(\mu_{i}-\mu\right)\left(\mu_{i}-\mu\right)^{T}
$$

and the within-class scatter matrix be defined as:

$$
S_{w}=\sum_{i=1}^{c}\left(X_{k}-\mu_{i}\right)\left(X_{k}-\mu_{i}\right)^{T}
$$

where $\mu_{\mathrm{i}}$ is the mean image of class $X_{i}$, and $N_{i}$ is the number of samples in class $X_{i}$. If $S_{w}$ is nonsingular, the optimal projection $W_{o p t}$ is chosen as the matrix with orthonormal columns which maximizes the ratio of the determinant of the between-class scatter matrix of the projected samples to the determinant of the within-class scatter matrix of the projected samples, i.e.,

$$
W o p t=\arg \max _{W} \frac{\left|W^{T} S_{B} W\right|}{\left|W^{T} S_{W} W\right|}=\left[w_{1}, w_{2}, \ldots \ldots, w_{m}\right] \ldots
$$

where $\left\{\mathrm{W}_{\mathrm{i}} \mid \mathrm{i}=1,2 \ldots \ldots \ldots, m\right\}$ is the set of generalized eigenvectors of $S_{B}$ and $S_{w}$ corresponding to the $m$ largest generalized eigenvalue $\quad\left\{\lambda_{i} \mid i=1,2, \ldots \ldots . m.\right\} \quad$ i.e., $S_{B} W_{i}=\lambda_{i} S_{W} W_{i}, i=1,2, \ldots \ldots . m$. There are at most $c-1$ nonzero generalized eigenvalues and so an upper bound on $m$ is $c-1$, where $c$ is the number of classes [11].

\subsection{Basic Steps Employed in FLD}

Step 1: The ratio of between-class and within-class scatter matrices is calculated.

Step 2: The eigenvectors of this matrix are then taken to formulate the projection matrix.

Step 3: The low dimensional sub-space created maximizes between-class scatter, while minimising within-class scatter.

\subsection{Fisherfaces Algorithm for FLD}

1. A Fisherfaces algorithm uses classes of images instead of just single images. A class in this instance is a type of object, for instance a barrel, a pizza, or a strawberry.

2. The system is trained with multiple images from each class, creating Eigenvectors similar to Eigenfaces, but attempts to maximize the differences between classes while minimizing differences within classes.

3. Classes are then projected into this space by projecting each image of the class and taking the average.

4. Input images can then be projected into Fisher space and compared to the average class projections.

\section{EXPERIMENTAL RESULTS}

In this research PCA and FLD methods for face recognition have been implemented and tested for a database size of 100 RGB color images. It has been observed that the recognition of the images is better for FLD (fisherface) than PCA (eigenface) based algorithm.

To test the robustness of this system, noise was added to the database images and set as trained database images. Salt and Pepper noise and Gaussian noise were considered as the noise model. Salt and pepper noise is a form of noise typically seen on images. This noise refers to single pixel noise statistics. It represents itself as randomly occurring white and black pixels. Also, to test the robustness, it rotated the images and placed the rotated images to the trained database. A sample test image considered for this experiment is shown in Fig. 1.

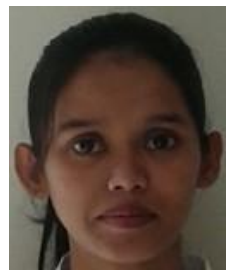

Fig. 1: A sample test image 
To test the robustness of the system, it rotated the image of Fig.1 by different angles (both clockwise and anticlockwise) and set as trained database images as shown in Fig. 2 and Fig. 3.

Table 1 shows the comparative performance of PCA and FLD methods for clockwise and anticlockwise rotated images.

Table 1. Performance for Rotational Situation

\begin{tabular}{|c|c|c|c|}
\hline $\begin{array}{c}\text { Rotated by } \\
\text { degrees }\end{array}$ & $\begin{array}{c}\text { Rotation } \\
\text { type }\end{array}$ & PCA & FLD \\
\hline $\begin{array}{c}1^{0,} 2^{0,} 3^{0, \ldots} \\
\cdots \cdots \cdots\end{array}$ & Clockwise & $\begin{array}{c}\text { Works well } \\
\text { upto: } 1^{0}\end{array}$ & $\begin{array}{c}\text { Works well } \\
\text { upto: } 9^{0}\end{array}$ \\
\hline $1^{0,} 2^{0,} 3^{0, \ldots}$ & $\begin{array}{c}\text { Anti- } \\
\text { clockwise }\end{array}$ & $\begin{array}{c}\text { Works well } \\
\text { upto: } 1^{0}\end{array}$ & $\begin{array}{c}\text { Works well } \\
\text { upto: } 12^{0}\end{array}$ \\
\hline
\end{tabular}

It is observed that FLD matches more degrees (for both clockwise and anticlockwise rotation) than PCA. Now Salt
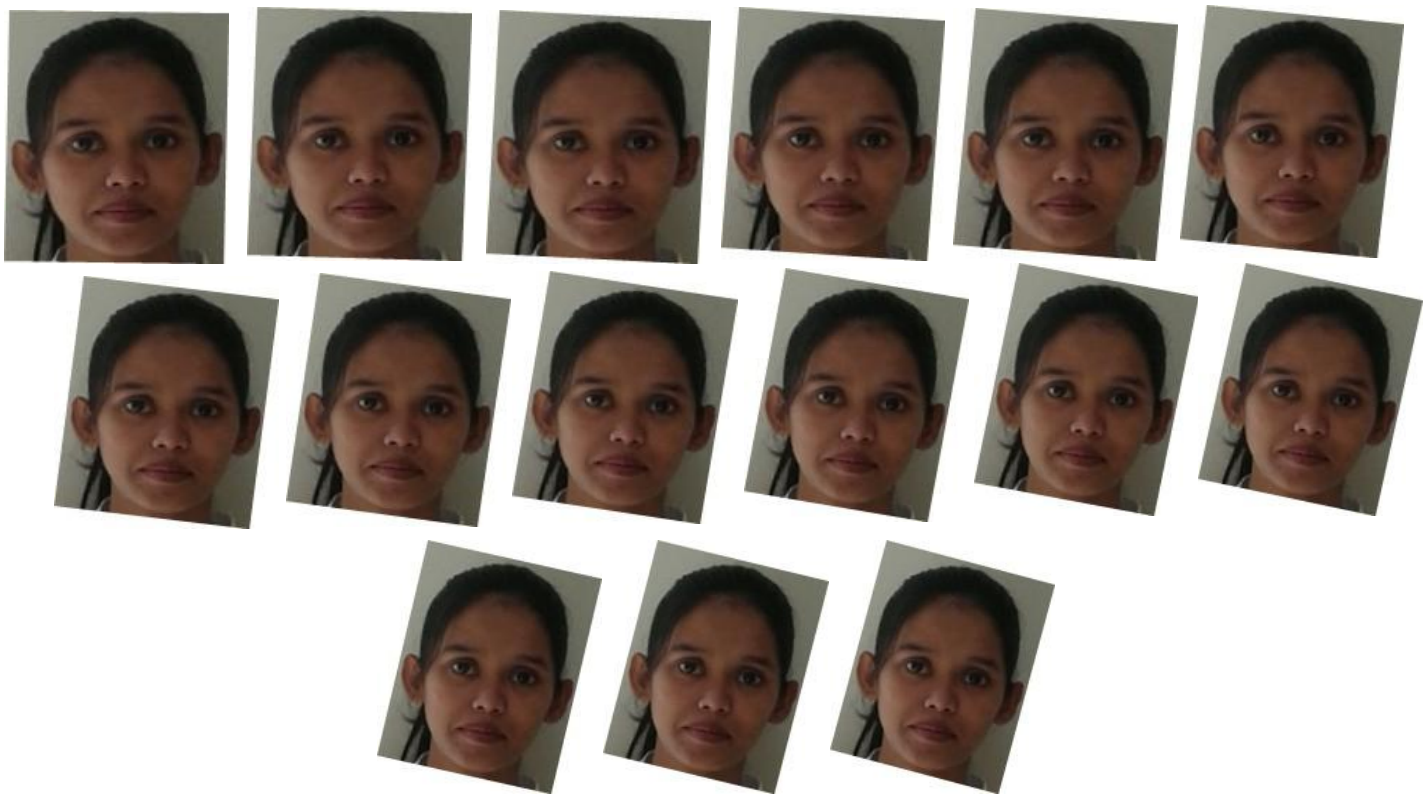

Fig. 2: Clockwise rotated by $1^{0}, 2^{0}, 3^{0}$
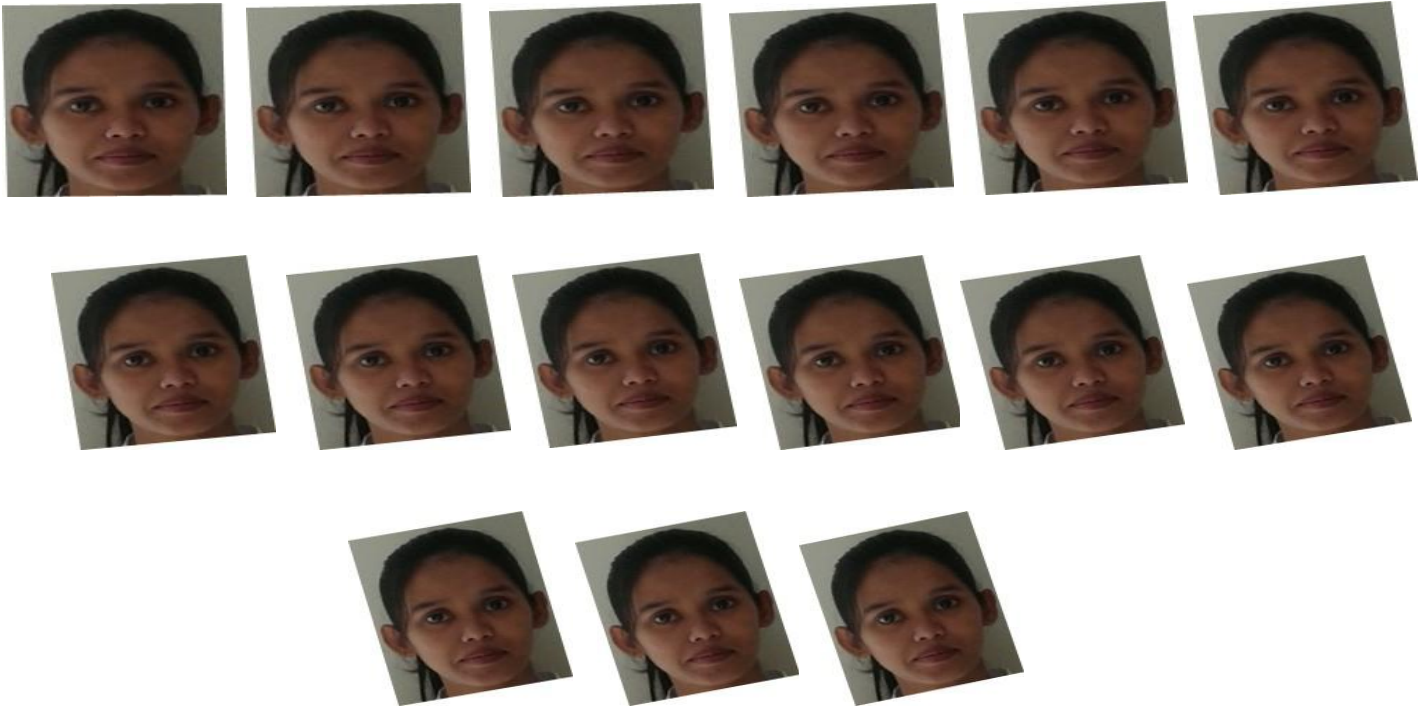

Fig. 3: Anticlockwise rotated by $1^{0}, 2^{0}, 3^{0} \ldots$ $15^{0}$ (from left to right) 

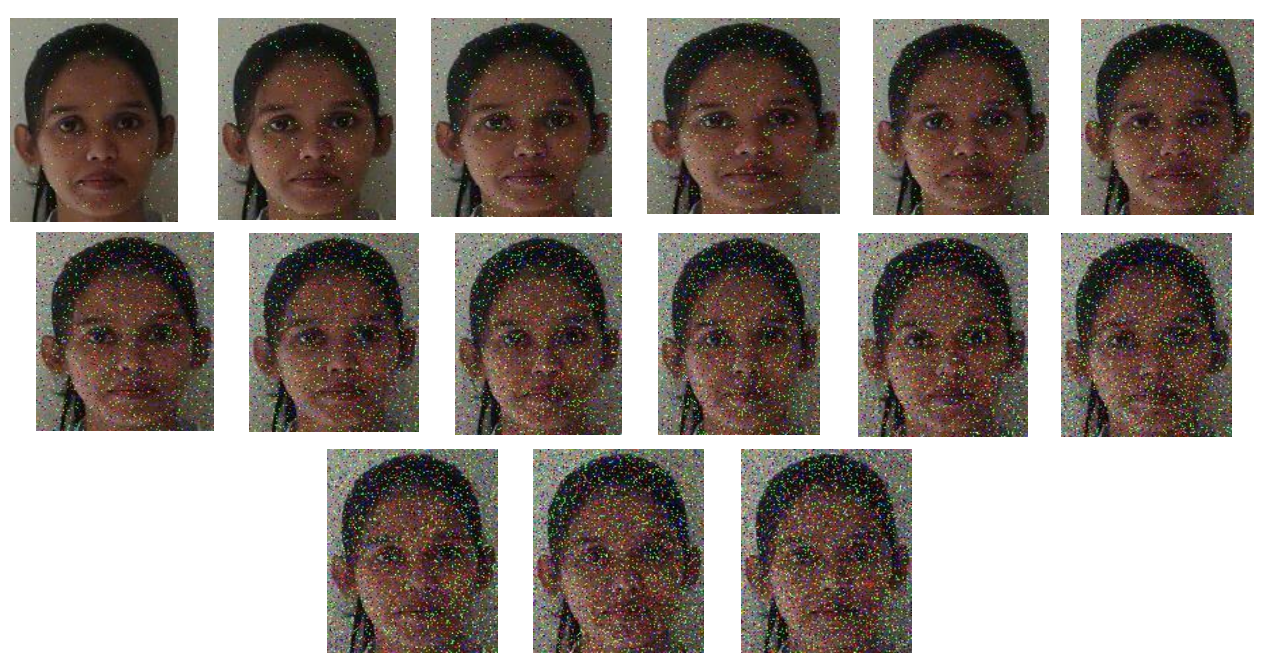

Fig. 4: Adding salt and pepper noise for density $d=0.01, d=0.02$, $\mathrm{d}=\mathbf{0 . 1 5}$ (from left to right)
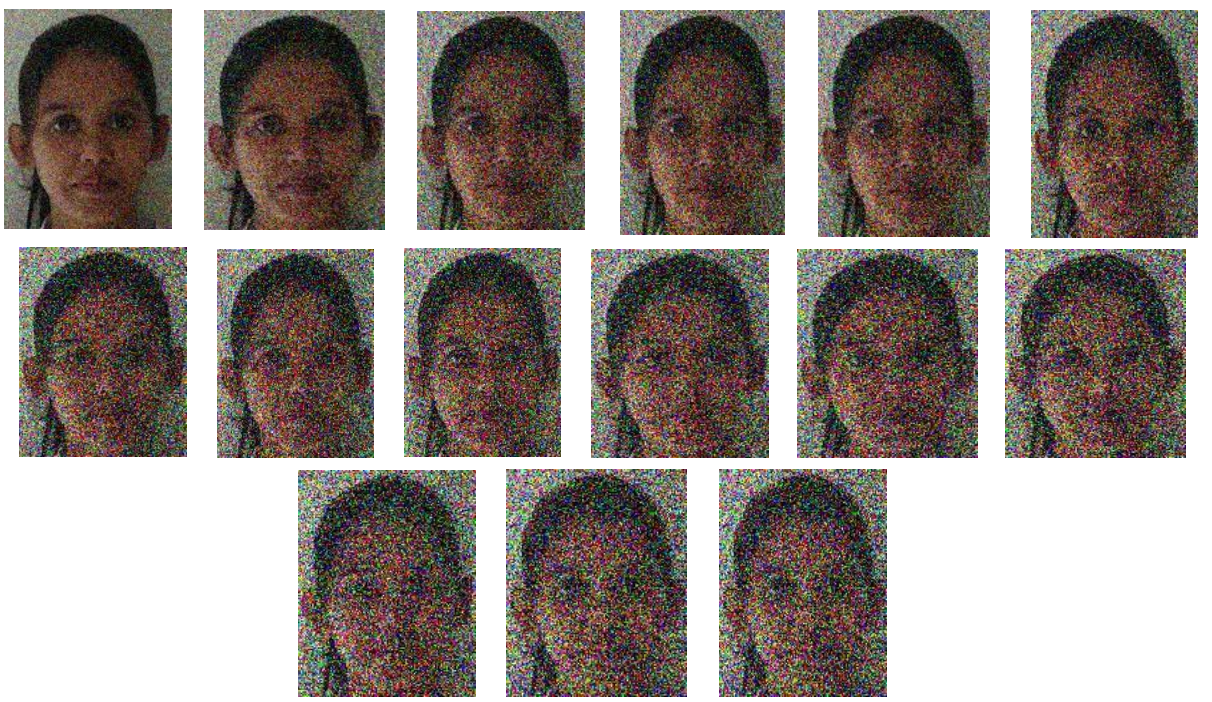

Fig. 5: Adding Gaussian noise (Mean=0, Variance $V=0.01,0.02,0.03, \ldots, 0.15$ )(from left to right)

Table 3. Performance for Noisy Situation for Gaussian Noise

\begin{tabular}{|c|c|c|c|}
\hline $\begin{array}{l}\text { Noise } \\
\text { Type }\end{array}$ & $\begin{array}{l}\text { Variance } \\
\text { (V) }\end{array}$ & PCA & FLD \\
\hline $\begin{array}{c}\text { Gaussian } \\
\text { noise }\end{array}$ & $\begin{array}{c}0.01,0.02, \\
0.03, \ldots \ldots \\
\ldots \ldots, 0.15\end{array}$ & $\begin{array}{c}\text { Works } \\
\text { well upto } \\
\text { variance, } \\
\mathrm{V}=0.01\end{array}$ & $\begin{array}{c}\text { Works well } \\
\text { upto } \\
\text { variance, } \\
\mathrm{V}=0.13\end{array}$ \\
\hline
\end{tabular}

\section{PERFORMANCE ANALYSIS}

PCA reduces the dimension size of an image greatly in a short period of time. It answers the question of which features are important for classification, and which are not. This analysis reduces the dimensionality of the training set, leaving only those features that are critical for face recognition.

FLD is similar to PCA but with improvement in better classification of different classes image. With FLD, the training set could be classified to deal with different people. Accuracy could has been better in the basis of noise and rotation than PCA approach. Besides, FLD removes the first three principal components.
FLD is more complex than PCA in finding the projection of face space. Calculation of ratio of between-class scatter to within-class scatter requires a lot of processing time. Besides, due to the need of better classification, the dimension of projection in face space is not as compact as PCA, results in larger storage of the face and more processing time in recognition.

While PCA maximizes for all the scatter as appropriate for signal representation, FLD differentiates between the withinclass scatter and between-class scatter as appropriate for pattern classification.

\section{CONCLUSION AND FUTURE WORK}

The measurement is done in MATLAB 7.8 and with Core i3 processor. Here, the performance measurement is categorized by two ways. Process time depends on image dimension and detection accuracy depends on image quality. In all cases, experiments have shown very promising results.

However, it did not has the chance of testing the algorithms for light sensitivity which is a major issue in computer vision. The lack of suitable databases with light variance was the problem. In future, implementation to test for light sensitivity would be the obvious step. The main issue in these algorithms 
would be the one of robustness versus simplicity. Due to the computational complexity of the FLD algorithm, it might be hard to implement in real time and given a large database, and a dense training set, pose sensitivity of the eigenface algorithm would not be significant. Hence, this would be a better choice of algorithm in most cases. But a possible combination of both algorithms which might include the simplicity of PCA and robustness of FLD would be a good step forward.

\section{REFERENCES}

[1] H. A. Rowley, S. Beluja, T. Kanade, "Neural NetworkBased Face Detection", IEEE Transactions on Pattern Analysis and Machine Intelligence, Vol. 20, No. 1, pp. 23-38, Jan. 1998

[2] R. Hsu. M. Mottleb, A. K. Jain, "Face Detection in Color Images", IEEE Transactions on Pattern Analysis and Machine Intelligence, Vol. 24, No. 5, May 2002.

[3] Y. Yokoo, M. Hagiwara, "Human Faces Detection Method using Genetic Algorithm", in Proc. IEEE Int. Conf. on Evolutionary Computation, pp. 113-118, May 1996.

[4] G. Yang, T. S. Huang, "Human Face Detection in a Complex Background", Pattern Recognition, Vol. 27, No. 1, pp. 53-63, 1994

[5] V. Perlibakas, "Face Recognition using Principal Component Analysis and Wavelet Packet Decomposition", Informatica, Vol. 15, No. 2, pp. 243250, 2004.

[6] K. Kim, "Face Recognition using Principal Component Analysis", National Institute of Technology, Rourkela, India, pp. 89-97, 2008.

[7] V. Perlibakas, "Distance Measures for PCA-based Face Recognition", Pattern Recognition Letters, Vol. 25, No. 6, pp. 711-724, April 2004.

[8] M. Turk, A. Pentland, "Face Recognition using Eigenfaces", Conference on Computer Vision and Pattern Recognition, 3 - 6 June 1991, Maui, HI , USA, pp. 586-591.

[9] H. Kong, L. Wang and E. K. Teoh, J.G. Wang and V. Ronda, "A Framework of 2D Fisher Discriminant Analysis: Application to Face Recognition with Small Number of Training Samples", IEEE Conference on Computer Vision and Pattern Recognition (CVPR), pp. 1083-1088, 2005.
[10] Harmon, L.D., "The recognition of faces", Scientific American, Vol. 5, pp. 71-82, 1973.

[11] P. N. Belhumeur, J. Hespanda, and D. Kiregeman, "Eigenfaces vs. Fisherfaces: Recognition Using Class Specific Linear Projection", IEEE Trans. on PAMI, Vol. 19, No. 7, pp. 711-720, July 1999.

\section{AUTHOR'S PROFILE}

Mahbuba Begum received her B.Sc. and M.S. degree in Computer Science and Engineering from Jahangirnagar University, Bangladesh, in the year 2007 and 2009, respectively. Currently, She is serving in the department of Computer Science and Engineering, Mawlana Bhashani Science and Technology University, Tangail, Bangladesh as a Lecturer. Her research interest is image processing, pattern recognition, face recognition $\&$ trademarks recognition.

Md. Golam Moazzam received his B.Sc. and M.S. degree in Computer Science and Engineering from Jahangirnagar University, Bangladesh. Currently, he is serving in the department of Computer Science and Engineering, Jahangirnagar University, Dhaka, Bangladesh as an Associate Professor. His research interest is image processing, pattern recognition, face recognition \& trademarks recognition.

Mohammad Shorif Uddin received his $\mathrm{PhD}$ in Information Science from Kyoto Institute of Technology, Japan, Masters of Education in Technology Education from Shiga University, Japan and Bachelor of Science in Electrical and Electronic Engineering from Bangladesh University of Engineering and Technology (BUET). He joined the Department of Computer Science and Engineering, Jahangirnagar University, Dhaka in 1992 and currently serves as a Professor of this department. His research is motivated by applications in the fields of computer vision, pattern recognition, blind navigation, bioimaging, 3D reconstruction, medical diagnosis and disaster prevention. He has published over 50 papers in peer-reviewed international journals and conference proceedings. He holds two patents for his scientific inventions. He received the best presenter award from the International Conference on Computer Vision and Graphics (ICCVG 2004), Warsaw, Poland, the best paper award in the International Conference on Informatics, Electronics \& Vision (ICIEV2013), Dhaka, Bangladesh. He is the co-author of two books. He is also a member of IEEE, SPIE, IEB and a senior member of IACSIT. 